\title{
A non-linear and non-iterative noise reduction technique for medical images: concept and methods comparison
}

\author{
Wilbur C.K. Wong ${ }^{\mathrm{a}}{ }^{*}$, Albert C.S. Chung ${ }^{\mathrm{a}}$ \\ ${ }^{a}$ Department of Computer Science, The Hong Kong University of Science and Technology, HK
}

\begin{abstract}
Filtering is a preliminary process in many medical image processing applications. It is aiming at reducing noise in images. Any post-processing tasks may benefit from the reduction of noise. In this contribution, a method for edge-preserving smoothing of 2D and 3D medical images is described. The proposed method uses a narrow spatial window and takes only a single iteration to denoise an image. It integrates geometric, photometric and local structural similarities to achieve non-linear noise reduction. We have applied this novel method to medical images and compared its denoising capability with other noise reduction techniques. The experimental results have shown that our method is capable of reducing severe noise, and is an adequate pre-process to improving the quality of segmentation and facilitating the feature extraction process.
\end{abstract}

Keywords: Non-linear filter; adaptive filter; smoothing method; biomedical image processing

\section{Introduction}

Filtering is a preliminary process in many medical image processing applications. It is a fundamental operation in low level computer vision, aiming at restoring a noisecorrupted image to its noiseless counterpart. Any post-processing tasks such as segmentation and feature extraction may benefit from the reduction of noise. Diffusion equations with scalar- and tensor-valued diffusivities have been applied to magnetic resonance imaging (MRI) and 3D rotational angiography (RA) [1,2,3] for edgepreserving smoothing. Magnetic resonance angiography (MRA) denoising with adaptive filtering in the Fourier domain has been proposed in [4]. Non-linear noise reduction techniques in computed tomography (CT) imaging have been investigated in [5].

In this paper, we present a novel non-linear filtering method for medical images, namely trilateral filtering (TF). Our method, which works along similar lines to bilateral filtering (BF) [6], integrates geometric, photometric and local structural similarities to achieve edge-preserving smoothing. The method is simple to implement and is applicable to multi-dimensional signals. It only uses a narrow spatial window (3 pixels in

\footnotetext{
* Corresponding author. E-mail address: cswilbur@cs.ust.hk.
} 
each dimension) and just takes a single iteration in the smoothing process (i.e. the method is non-iterative). The experiments on digital subtraction angiography (DSA) and MRI have shown that our new filtering technique TF is capable of reducing noise (even severe noise) in medical images. Post-processing tasks, for instance, segmentation and feature extraction on the filtered images, have demonstrated the competence of TF in improving the quality of segmentation and in facilitating the feature extraction process.

\section{Concept}

Bilateral filtering $(\mathrm{BF})$, representing a large class of non-linear filters proposed by Tomasi and Manduchi [6], is a non-iterative and local approach to edge-preserving smoothing. A filtered image is obtained by replacing the intensity value of each pixel with an average value weighted by the geometric and photometric similarities between neighboring pixels within a spatial window. The concept of BF has been investigated in a recent publication. Barash [7] revealed that bilateral filtering is non-iterative only if a wide spatial window is used (15 pixels in each dimension). However, a wide spatial window may over-smooth sharp ridges and gutters in the image as indicated in [8].

Sharp ridges and gutters are commonly found in medical images, such as nested vessels in DSA, and folded white matter (WM) and gray matter (GM) in brain MRI. Therefore, a narrow spatial window, say, 3 pixels in each dimension, should be used in order to avoid over-smoothing structures of sizes comparable to the image resolutions. This leads to the necessity of performing more iterations in the filtering process with BF. As such, it is required to strike a balance between the size of the spatial window and the number of iterations needed to be performed in BF. To address this limitation, we propose a novel filtering technique for medical images. Our method aspires to filter the images with a narrow spatial window in a single iteration while preserving the sharp ridges and gutters.

\section{Novel Method}

The newly proposed method works along similar lines to BF. Nonetheless, it not only takes the geometric and photometric similarities into account, but also makes use of the local structural similarity to smooth the medical images. Local structural information is used to determine inhomogeneity in the images. On one hand, low-pass filtering is performed in the homogeneous regions. On the other hand, smoothing along edges is achieved by considering the geometric, photometric and local structural similarities between neighboring pixels in the inhomogeneous regions. Because the three similarities are used in the filtering process, we name this novel method trilateral filtering (TF).

The local structural information is obtained from the eigen decomposition of orientation tensors as described in [9]. The Frobenius norm of the orientation tensor defines the local signal amplitude. It is used to determine inhomogeneity in the images. 
While the eigenvectors, corresponding to the first and second smallest eigenvalues in the decomposition, depict the direction of minimum and maximum curvatures respectively. They are employed in the formulation of the local structural similarity. Local structural similarity is aimed at regulating the filtering process. Together with the geometric and photometric similarities, filtering along the orientation of the ridges and gutters can be achieved, whereas low-pass filter is applied to the homogenous regions. For detailed formulation of the methodology, see our related work [10].

\section{Results and methods comparison}

We have applied the novel non-linear filtering technique TF to $2 \mathrm{D}$ and 3D medical images, and compared its denoising capability with Gaussian filtering (GF), BF and edge-enhancing anisotropic diffusion (EED) [3]. In all the experiments, a narrow spatial window ( 3 pixels in each dimension) is used in TF, GF and BF, and the filters are applied once (i.e. a single iteration is executed) on the images.

\subsection{D medical image}

A 2D DSA image has been tested with TF, GF and BF. Results are shown in Fig. 1. It is observed that the background noise is reduced significantly with all the three methods. In visual inspections of the filtered images, $\mathrm{TF}$ presents the best edge-preserving denoising capability. It is found that, in the TF-filtered image, the vessels are smoothed along the vascular boundaries, as opposed to the blur boundaries appear in the GFfiltered image; whereas BF introduces severe artifacts at the boundary of vessels, on account of depending solely on the geometric closeness and photometric similarities in the filtering process (see the areas highlighted by the arrows).

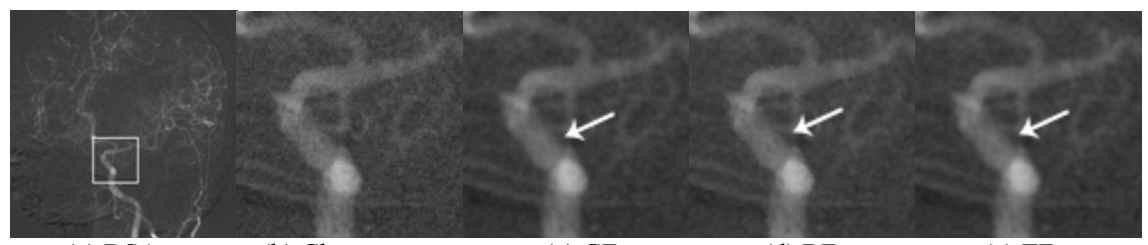

(a) DSA

(b) Close-up

(c) GF

(d) BF

(e) $\mathrm{TF}$

Fig. 1. DSA image and its filtered images. (a) DSA image; (b) close-up of the image; (c) GF-filtered image; (d) BF-filtered image; and (e) TF-filtered image.

Given manual segmentation of the DSA image, we have calculated the contrast-tonoise ratio (CNR) of the noisy image and its filtered counterparts. CNR is defined as the squared ratio of the difference in the sample mean intensity values of the vessel (i.e. object of interest in general) and the background to the sample standard deviation of the background. Table 1 lists the CNR of the noisy DSA image and its filtered images. It 
reveals that TF provides the largest increase $(>50 \%)$ in $\mathrm{CNR}$, as compared to the other two filters.

Table 1

CNR of the noisy DSA image and its filtered images.

\begin{tabular}{|c|c|c|c|c|}
\cline { 2 - 5 } \multicolumn{2}{c|}{ Noisy } & GF-filtered & BF-filtered & TF-filtered \\
\hline CNR & 12.15 & 17.64 & 17.25 & 18.45 \\
\hline
\end{tabular}

In further studies, a double-Gaussian mixture model [11] has been used to segment the aforesaid filtered images. The segmented images are shown in Fig. 2(a)-(c). It is noted that the delineation of the vascular region in the segmentation of the BF-filtered image are not as smooth as the segmentations of the TF-filtered and GF-filtered images, where most of the vessels are connected with smooth delineation and very few background pixels are misclassified. In addition to segmentation, we have applied Roberts cross operator [12] to detect edges in the filtered images. Roberts cross operator is employed for comparing the quality of filtering because of its high sensitivity towards noise in detecting edges and weak response to genuine edges (unless they are very sharp). In this work, the edge maps produced by the Roberts cross operator are used to justify the edgepreserving denoising capability of the filters. In Fig. 2(d)-(f), it is observed that the edge map produced with the TF-filtered image contains the least amount of false edges while most of the detected edges are connected, as compared to the edge map of the GFfiltered and BF-filtered images.

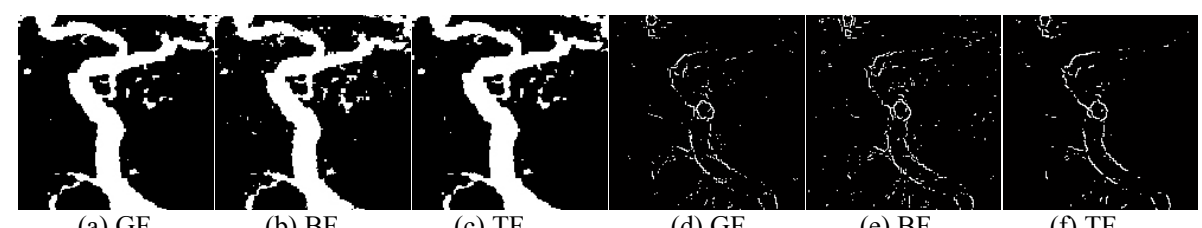

(a) GF (b) $\mathrm{BF}$

(c) TF

(d) $\mathrm{GF}$

(e) $\mathrm{BF}$

(f) $\mathrm{TF}$

Fig. 2. DSA segmented images and edge maps. (a) Segmented GF-filtered image; (b) segmented BF-filtered image; (c) segmented TF-filtered image; (d) edge map of the GF-filtered image; (e) edge map of the BFfiltered image; and (f) edge map of the TF-filtered image.

\subsection{D medical image}

For the experiments with 3D medical images, an MRI volume of normal brain from the BrainWeb is tested. The volume is of noise level 9\% (the highest noise level available) without any intensity non-uniformity. We have tested the MRI volume with $\mathrm{TF}, \mathrm{GF}, \mathrm{BF}$ and EED. It is noticed that, in visual inspections of the filtered images (see Fig. 3), TF produces good restoration of the noisy volume, contrary to BF that produces the worst denoised image amongst all the other methods. While the filtered images obtained by GF and EED appear to be over-smoothed across different anatomy boundaries, especially boundary between WM and GM. Table 2 lists the CNR of WM 
and GM in the noisy MRI volume and its filtered images. These figures indicate that TF provides the largest increase in the CNRs of WM and GM, as opposed to BF which provides the smallest increase in those CNRs. These findings accords with the observation in the aforementioned visual inspections.

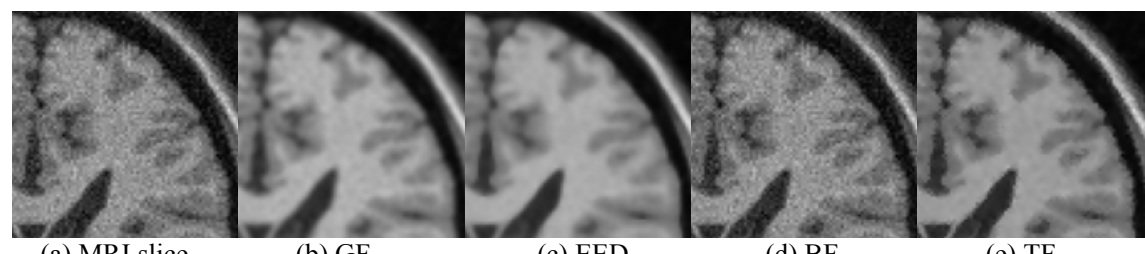

(a) MRI slice

(b) GF

(c) EED

(d) BF

(e) $\mathrm{TF}$

Fig. 3. MRI slice image and its filtered images. (a) Slice image; (b) GF-filtered image; (c) EED-filtered image; (d) BF-filtered image; and (e) TF-filtered image.

Table 2

CNR of the white matter (WM) and gray matter (GM) in the noisy MRI volume and its filtered images.

\begin{tabular}{|c|c|c|c|c|c|}
\cline { 2 - 6 } \multicolumn{1}{c|}{} & Noisy & GF-filtered & EED-filtered & BF-filtered & TF-filtered \\
\hline CNR of WM & 200.80 & 1526.16 & 1607.81 & 209.44 & 1661.73 \\
\hline CNR of GM & 104.93 & 816.47 & 882.98 & 109.47 & 895.31 \\
\hline
\end{tabular}

In a further study of the segmentation ${ }^{1}$ of the filtered images (see Fig. 4 for the results), we have found that the segmentation of the TF-filtered image is the most adequate amongst all its counterparts. This result is expected because TF has a good image restoration capability comparing to the other methods, as has been shown in the previous experimental results.
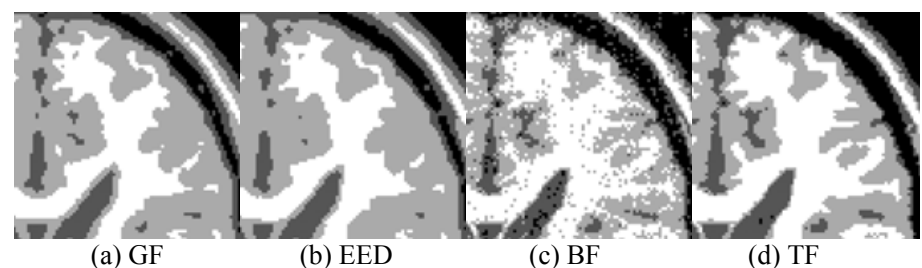

(c) $\mathrm{BF}$

(d) $\mathrm{TF}$

Fig. 4. Segmented images of the filtered MRI slice image. (a) Segmented GF-filtered image; (b) segmented EED-filtered image; (c) segmented BF-filtered image; and (d) segmented TF-filtered image.

\section{Conclusion}

We have presented a novel denoising method for medical images, namely the trilateral filter. The method integrates the geometric, photometric and local structural similarities to filter medical images. It replaces the intensity value at each pixel with an average

\footnotetext{
${ }^{1}$ The segmentation is performed by adopting the algorithm proposed in [13]. We assume there are four classes in the MRI volume, viz. background, cerebral spinal fluid (CSF), gray matter (GM) and white matter (WM).
} 
value weighted by the three similarities between neighboring pixels within a spatial window. Our method is simple to implement and is applicable to multi-dimensional images. Furthermore, it uses a narrow spatial window and takes only a single iteration to smooth an image while preserving the edges.

In order to evaluate the performance of the trilateral filter, we have conducted several experiments on 2D and 3D medical images. The experimental results have shown that our novel method is capable of producing greater noise reduction and smoothing the images without over-smoothing the edges, as compared to other edge-preserving noise reduction methods and the Gaussian filter. Additionally, the application of the postprocessing tasks - segmentation and feature extraction (edge detection) - on the filtered images has demonstrated that the trilateral filtering is an adequate pre-processing technique to improving the quality of segmentation and facilitating the feature extraction process.

\section{References}

[1] Gerig G, Kübler O, Kikinis R, Jolesz FA. Nonlinear anisotropic filtering of MRI data. TMI 1992: 11(2):221-32.

[2] Krissian K. Flux-based anisotropic diffusion applied to enhancement of 3-D angiogram. TMI 2002: 21(11):1440-2.

[3] Meijering E, Niessen W, Weickert J, Viergever M. Diffusion-enhanced visualization and quantification of vascular anomalies in three-dimensional rotational angiography: Results of an in-vitro evaluation. MedIA 2002: 6(3):215-33.

[4] Westin CF, Wigström L, Loock T, Sjöqvist L, Kikinis R, Knutsson H. Three-dimensional adaptive filtering in magnetic resonance angiography. JMRI 2001: 14:63-71.

[5] Fernández G, Bischof H, Beichel R. Nonlinear filters on 3D CT imaging — bilateral filter and mean shift filter. In: Proceedings of the Computer Vision Winter Workshop; 2003. p. 21-6.

[6] Tomasi C, Manduchi R. Bilateral filtering for gray and color images. In: Proceedings of the ICCV; 1998. p. 839-46.

[7] Barash D. A fundamental relationship between bilateral filtering, adaptive smoothing, and the nonlinear diffusion equation. PAMI 2002: 24(6):844-7.

[8] Choudhury P, Tumblin J. The trilateral filter for high contrast images and meshes. In: Proceedings of the Eurographics Symposium on Rendering; 2003. p. 186-96.

[9] Knutsson H. Representing local structure using tensors. In: Proceedings of the $6^{\text {th }}$ Scandinavian Conf. on Image Analysis; 1989. p. 244-51.

[10]Wong WCK, Chung ACS, Yu SCH. Trilateral filtering for biomedical images. In: Proceedings of the ISBI; 2004. To appear.

[11] Jin JS, Wang Y, Hiller J. An adaptive nonlinear diffusion algorithm for filtering medical images. IEEE Trans. Info. Tech. Biomed. 2000: 4(4):298-305.

[12]Roberts LG. Machine perception of 3-D solids. In: Optical and Electro-optical Information Processing. MIT Press; 1965. p. 159-97.

[13]Fwu JK, Djurić PM. Unsupervised vector image segmentation by a tree structure - ICM algorithm. TMI 1996: 15(6):871-80. 